\title{
Über die Schwierigkeiten der Pädagogik, über Leistung und Gerechtigkeit im Schulsystem zu reden. Eine Metakritik zu Berkemeyers Nerowski-Kritik
}

\author{
Heinz-Elmar Tenorth
}

Online publiziert: 26. Februar 2020

(C) Der/die Autor(en) 2020

Das war natürlich zu erwarten, dass der Versuch von Christian Nerowski (2018), über Leistung als Kriterium schulischer Bewertung und Zertifizierung auch gerechtigkeitstheoretisch zu argumentieren, auf Widerspruch stoßen würde. Nils Berkemeyers Kritik (Berkemeyer 2018) reiht sich insofern zunächst nur in die bekannte Traditionslinie ein, in der innerhalb der historischen Reformpädagogik früh, in der bildungstheoretisch fundierten Literatur erneut und argumentprägend (z. B. Klafki 1991) und in der kontroversen Debatte über Bildungsstandards und Disparitäten in Bildungskarrieren und Lebensläufen erst jüngst, aber immer heftig über Leistung gestritten wurde. Berkemeyer nimmt Nerowskis Neueinsatz in dieser alten Frage allein insofern ernst, als er die gerechtigkeitstheoretische Begründung ins Zentrum rückt, hier allerdings schweres Geschütz auffährt und an Nerowski kein gutes Haar lässt, verbunden mit knappen Bemerkungen zu dessen schultheoretischen Prämissen, für die er, wie man auch erfährt, ebenfalls wenig übrighat.

Die Kritik stützt sich indes, betrachtet man sie näher, auf Argumente, die es nicht empfehlenswert erscheinen lassen, mit Berkemeyer zur alten Linie zurückzukehren und Leistung jetzt auch gerechtigkeitstheoretisch als Kriterium im (schul-)pädagogischen Kontext definitiv ad acta zu legen. Berkemeyers Kritik scheint das nahezulegen, aber sie ist nicht so fundiert, dass man ihr folgen sollte. Das gilt, wie ich im Folgenden zeigen will, zuerst für den Vorwurf, ein Problem zu bearbeiten, das längst gelöst ist, das gilt, ferner, für seine Behauptung, Nerowski habe die gerechtigkeitstheoretische Diskussionslage falsch, verkürzt und nicht problemangemessen rezipiert, und es gilt schließlich, drittens, für seine Kritik an den schultheoretischen Annahmen in Nerowskis Analyse. Ihrer Struktur nach, das ist die systematische

Prof. i.R. Dr. H.-E. Tenorth ( $₫)$

Abteilung Historische Bildungsforschung, Humboldt-Universität zu Berlin, Unter den

Linden 6, 10099 Berlin, Deutschland

E-Mail: tenorth@hu-berlin.de 
Implikation meiner Metakritik, machen Nerowski und Berkemeyer aber gemeinsam und zuerst bewusst, dass der Rekurs auf Leistung und die Zertifizierungs- und Selektionsfunktion von Schule innerhalb der Pädagogik neu behandelt werden muss und dass dabei auch die Bedeutung allgemeiner Gerechtigkeitstheorien im spezifischen Feld von Pädagogik und Schule zum Thema werden sollte. Wird Leistung legitimierbar zu einem Kriterium schulischer Arbeit, sind die Folgeprobleme größer als der gerechtigkeitstheoretische Streit sichtbar macht. Die Begründung dieser Metakritik mündet deshalb in knappe Hinweise zu den offenen Fragen, die beide Autoren hinterlassen und die jenseits der Grundlagendebatte im pädagogischen Kontext besonders dringlich gestellt und zu lösen sind.

\section{Leistung als kriteriales Problem in pädagogischer Praxis - ein Systemproblem pädagogischer Reflexion}

Berkemeyer geht eingangs in seiner Kritik davon aus, dass Nerowski mit seiner Argumentation ,ein Ziel verfolgt ..., welches bereits realisiert ist und ihre Grundidee bereits in Walzers Hauptwerk ,Sphären der Gerechtigkeit' (2006 [1992]), dessen englischsprachiges Original 1983 erschien, formuliert worden ist, ohne dass aber auf Walzer Bezug genommen wird." Nerowski mühe sich also - unnötigerweise - an einer Rehabilitierung von „Leistung“ ab, die doch gar nicht strittig, sondern Alltag sei. Für einen Kritiker, der so stark darauf pocht, dass die themenbezogene Diskussionslage nicht nur angemessen, sondern auch umfassend berücksichtigt werden soll, überrascht diese These, ignoriert er doch die breite Kritik, die das Leistungsprinzip im Allgemeinen und vor allem im pädagogischen Milieu erfährt. Das gilt ja nicht erst aktuell, wenn die damit exemplarisch verbundene Praxis der Bewertung individueller Schulleistungen primär nach curricularen Bezugsnormen diskutiert wird (z. B. Bohnsack 2013) oder wenn universale Kriterien im Kontext der Debatten über Bildungsstandards problematisiert und allein individuelle Bezugsnormen bildungstheoretisch als legitim behauptet werden (z. B. Herrmann 2004, 2011), weil es „Die Würde des heranwachsenden Menschen .. (aus) macht, sein eigener ,Standard“ sein zu dürfen." (Thurn 2004).

Alle diese Debatten bilden daher zu Recht den Ausgangspunkt bei Nerowski, den er auch systematisch, wie seine ausführlichen Belege gleich zu Beginn zeigen, als Stand der Diskussion dokumentiert. „Leistung“ ist im pädagogischen Diskurs ein Problem, die allgegenwärtige Praxis der Bewertung und Zertifizierung ist zwar gegeben, aber offenbar nicht systematisch begründet, jedenfalls nicht gegen Einwände immun. Die Frage aufzuwerfen, ob es Legitimation für diese Praxis gibt, ist deshalb auch sinnvoll, sie gerechtigkeitstheoretisch ohne Vorabverdikt aufzunehmen zwar in der Pädagogik nicht alltäglich, aber systematisch notwendig und aktuell gut begründet.

Liegt aber, so der systematische Einwand gegen Nerowski, in dessen gerechtigkeitstheoretischen Argumentation ,eine Form des Sein-Sollen-Fehlschlusses vor“, wie Berkemeyer sagt? Hier liegt für mich zunächst nur eine mutwillige Verzerrung des bei Nerowski zu diskutierenden Problems und seiner Konstruktion vor. Denn keineswegs argumentiert er so, wie Berkemeyer suggeriert, und er geht auch nicht 
von einer „Präzisierungsannahme“ aus. Er markiert vielmehr Begründungsbedarf, und er legt auch nicht nahe, dass ,aus der Beschreibung des Sachverhalts der Selektion“ folge, „dass man diese nur durch das Leistungsprinzip, ohne Angabe von Verfahren, gerecht lösen kann. “Schon das „nur“ findet sich nirgendwo bei Nerowski, hier ist Leistung nur „,ein Kriterium“ (Nerowski, Herv. HET) und hier werden „die institutionellen Setzungen des Schulsystems nicht als invariant betrachtet", sondern allenfalls als gegeben, und gefragt, ob das Gegebene der Rechtfertigung zugänglich ist, und zwar jenseits der Normativität des Faktischen oder der Varianz der Realisierung. In seiner Problemformulierung liefert Berkemeyer mit seiner Kritik deshalb nur einen Beweis für die gut bekannte pädagogische These, dass dem Gegebenen keine Legitimation zukommen kann, getreu dem für kritische Pädagogen offenbar klassisch gewordenen Argument Klaus Mollenhauers, dass „das vergesellschaftete Dasein immer schon ein defizienter Modus der Möglichkeiten des Menschen ist“, und zwar deswegen, weil ,die gesellschaftlichen Implikationen des Heranwachsens prinzipiell dasjenige reduzieren, was als Mündigkeit doch die erklärte Norm dieses Vorgangs sein sollte." (Mollenhauer 1964, S. 65) Schließlich und nur als kleine Randbemerkung: Ist das Argument pro Leistung ein Argument, das man als „moralisch" qualifizieren, begründen oder kritisieren kann, oder nicht doch eher als „ethisch“, nämlich als Frage nach der Rechtfertigung von Praktiken und Moralen durch Prinzipien, wie Berkemeyer an anderer Stelle ja selbst unterscheiden kann, um dann die Praktiken in seiner Argumentation - wie Nerowski auch - selbst zu ignorieren und sich auf die gerechtigkeitstheoretische Debatte zu stürzen. Verdient seine Argumentation aber hier mehr Kredit, trotz der problematischen Ausgangskonstellation?

\section{Gerechtigkeitstheorien im spezifischen Feld der Pädagogik/der Schule}

Die gerechtigkeitstheoretische Debatte, wie Berkemeyer sie fortsetzt, bestätigt erneut eine Erfahrung, die man aus der Pädagogik und ihren Kontroversen sehr gut kennt, und sie bestätigt damit ebenfalls die geringe Fruchtbarkeit solcher Argumentationsstrategien. Auch seine Kritik lebt wesentlich von vermeintlich systematisch relevanten Rezeptionslücken oder falscher Rezeption, erzeugt also im Wesentlichen eine exegetische Debatte über die richtige oder falsche Lesart der Theoretiker, die Nerowski nutzt bzw. ignoriert. Unfruchtbar ist diese Strategie, weil Berkemeyer jeweils nur knapp seine eigene Lesart von Theorien andeutet, die selbst viel komplexer sind, für die er aber dennoch mutig und urteilsfreudig Lücken oder Rezeptionsfehler konstatiert. Diesen Befund will ich deshalb auch nicht weiter im Detail diskutieren und etwa zeigen, wie selektiv - notwendiger Weise und unvermeidlich - Berkemeyer selbst verfährt, z. B. im Blick auf Taylor oder Rawls oder Honneths Anerkennungstheorie und ihre Diskussion in der Pädagogik (nur darauf komme ich später zurück). Auch seine Kritik an der „selektiven Verwendung von Theorieelementen“ ist nicht gegen Kritik immun, selbst wenn man die Praxis der Eklektik in der Pädagogik als strukturelles Problem schon historisch gut kennt. Aber spätestens im Alltag pädagogischer Arbeit, wenn nicht nur Prinzipien, sondern Praktiken zur Diskussion stehen, 
kann man auch systematisch nicht ignorieren, dass Prinzipien allein nicht reichen und die eklektische Nutzung von Argumenten und Prämissen unausweichlich wird.

Jenseits dieser systematischen Probleme will ich mich im Folgenden nur auf einen für ihn offenbar so zentralen wie für die Kritik an Nerowski scheinbar schlagenden Befund konzentrieren, die Nichtberücksichtigung der Arbeiten von Michael Walzer, mit denen Nerowskis Thema längst hinreichend bearbeitet sei. Ist das so, hat Walzer schon alle Probleme bereits gelöst ${ }^{1}$ und Nerowski nur einen Beleg dafür geliefert, das Nichtlesen Probleme erzeugt, die längst geklärt sind? Schaut man sich bei den Argumenten von Walzer und in ihrer Diskussion um, dann ist die Lage wohl eher anders. Nerowski, das ist meine Gegenthese, nimmt ein Problem auf, dass Walzer ebenfalls aufgeworfen, aber nicht hinreichend bearbeitet oder sogar ungelöst gelassen hatte und geht von einem aktuellen Kontext aus, der bereits jenseits von Walzer diskutiert und ein eigenes gerechtigkeitstheoretisches Angebot für die Legitimation von schulischen Lernprozessen und die dabei unausweichlichen Fragen nach Gleichheit und Gerechtigkeit und der Konstruktion von Differenz entwickelt hat.

Walzer, um diese von Berkemeyer mit einem starken Argument genutzte Referenz etwas näher einzuführen, hat in den ,Sphären der Gerechtigkeit“" gegen die dominierende prinzipientheorethische Argumentation seit Rawls die These entwickelt, dass „dass die Prinzipien der Gerechtigkeit in ihrer Form selbst pluralistisch sind“, so „dass verschiedene soziale Güter aus unterschiedlichen Gründen von verschiedenen Instanzen und gemäß unterschiedlicher Verfahren verteilt werden sollten; und dass sich alle diese Unterschiede aus den verschiedenen Verständnissen (understandings) der sozialen Güter selbst herleiten - dem unvermeidlichen Produkt eines geschichtlichen und kulturellen Partikularismus“ (Walzer 1983, S. 6; zit. nach Knoll und Spieker 2014, S. 15²). „Komplexe Gleichheit“" in der „Pluralität“ der Sphären und der Kriterien der Verteilung der jeweiligen Güter ist deshalb sein Thema, und ,ein Bildungszeugnis“ zählt zu den Exempeln solcher Güter, die Walzer schon in der Einführung bemüht, um sein Thema zu erläutern. Für Bildung und Schule schlägt Walzer also sphärenspezifisch eine Lösung vor, die sequentiell das Bedürfnis- und das Leistungsprinzip nutzt.

Walzer unterscheidet dabei sehr scharf zwei unterschiedliche Kriterien der Verteilung von Bildung (Walzer 2006 [1992], S. 288 ff.), die durch die Schule in ,relativer Autonomie" sowie auf Individuen und die soziale Reproduktion zugleich bezogen realisiert werden (Walzer 2006 [1992], S. 289). Sie muss sich dabei für die Lernenden biographisch, in der „Elementarerziehung“, zuerst vor der Erwartung der

\footnotetext{
1 Dabei kann ich, nur nebenbei, allerdings mein Erstaunen nicht unterdrücken, dass z. B. auch in Manitius et al. (2015) weder einleitend noch z.B. in dem systematischen Kapitel von Wigger (2015) - oder in den anderen systematisch argumentierenden Texten - die Theorie von Walzer oder ihre Diskussion überhaupt Eingang finden, wenn sie doch so zentral ist.

2 Ich zitiere diese Übersetzung nur, um die erste Schwierigkeit einer Rezeption zu bezeichnen, die z. B. Knoll und Spiecker angesichts ihrer Kritik an der Übersetzung der deutschen Ausgabe signalisieren. Dort heißt diese Passage: „daß die Prinzipien der Gerechtigkeit ihrerseits in ihrer Form selbst pluralistisch sind“ und weiter ,,daß die verschiedenen Sozialgüter aus unterschiedlichen Gründen von unterschiedlichen Agenten und Mittlern auf der Basis unterschiedlicher Verfahren verteilt werden sollten und daß alle diese Unterschiede sich herleiten aus den unterschiedlichen Bedeutungen der Sozialgüter selbst - dem unvermeidbaren Resultat eines historischen und kulturellen Partikularismus“". (so Walzer dt. 2006 [1992], S. 30) Systematisch kann ich das hier auf sich beruhen lassen.
} 
„einfachen Gleichheit“, d.h. auch „gemeinsame Arbeit für ein gemeinsames Ziel“ (Walzer 2006 [1992], S. 300) rechtfertigen. In der Nachfolge der in den USA dominierenden Schultheorie und Bildungssoziologie liefert die einheitliche Konstruktion des demokratischen Bürgers dafür die politisch-soziale Referenz und Begründung. Für die späteren Phase der Arbeit von Schulen ,verliert die einfache Gleichheit ihren Sinn“ (Walzer 2006 [1992], S. 301), dann zählen die Differenzen der Neigungen und der Leistung sowie die je individuellen und systemischen Erwartungen an differente Bildungs- und Berufskarrieren, bei Annahme der Zertifizierung als Form der Verteilung und unter ausdrücklicher Betonung der je individuellen „Leistung“ als Kriterium (Walzer 2006 [1992], S. 301 f.). Das auf die Individuen egalitär zugeschnittene Bedürfnis- und das universal geltende Leistungsprinzip werden also klar unterschieden und sequenziert, genauso wie Nerowski es auch tut.

Diese Position von Walzer ist, ebenfalls erwartbar, nicht unkritisiert geblieben, auch unter verteilungstheoretischen Kriterien, scheinen damit doch Privilegen der sozialen Herkunft vom Bildungssystem ungefiltert und wenig kompensiert nach der Elementarerziehung wieder zur Geltung zu kommen, u. a. vom Leistungsprinzip scheinbar legitimiert (Schwaabe 2014). Nerowski nimmt zwar nicht die nach Walzer daraufhin entbrannte und walzer-bezogene Diskussion unmittelbar auf, aber seine Referenzen und Unterscheidungen gehen von den späteren Versuchen aus, Egalität und Differenz, Gleichheit und Gerechtigkeit im Bildungssystem zugleich zu befördern und ihre Vereinbarkeit und Einlösbarkeit zu rechtfertigen. Wie Walzer sieht er dabei mit seinen Gewährsmännern Lösungen in der Sequenzierung von Praxen und Kriterien, Gleichheit für die Phase des Bildungsminimums, zunehmende Akzeptanz von Differenz und individuelle Zurechnung von schulisch erbrachten Leistungen danach, und legitime Zuweisung von Zertifikaten als den erstrebten Gütern in der zweiten Phase nach Leistungskriterien. Für diese Arbeit am Thema der „komplexen Gleichheit“ bemüht Nerowski - ohne Walzer explizit zu bemühen eine Diskussion, die inzwischen erziehungstheoretisch u. a. bei Giesinger, und sozialphilosophisch, u.a. bei Gosepath, auch mit internationaler Referenz und sehr selbstständig bestritten wird. Berkemeyer geht auf diese Diskussion nicht ein, mit deutlichen Folgen für die Geltung seiner Argumente z.B. über die Sequenz von Bildungsminimum und weiterer schulischer Bildung. Anders als in der erwähnten Diskussion unterscheidet er schon Zertifikate und Kompetenzen, Schulstrukturen und Bildungslaufbahnen als differente Referenzen für Gleichheits- und Gerechtigkeitserwartungen nicht so deutlich, wie das notwendig wäre. Selbst seine so radikal klingende, als alternativer Ausblick angekündigte Forderung - „In einer solchen Perspektive wäre dann auch anerkannt, dass Schülerinnen und Schüler einen Anspruch gegenüber der Schule haben." - findet sich explizit in den Begründungen für das Bildungsminimum, wenn es als „Bringeschuld der Schule“ interpretiert wird. Berkemeyer zeigt auch hier nur, dass er sowohl hinter Walzer zurückbleibt als auch hinter den bildungstheoretischen Erläuterungen, in denen in der deutschen Diskussion zur Präzisierung des Sinns und der Möglichkeiten von allgemeiner Bildung, aber äquivalent zur Unterscheidung von ,einfacher Gleichheit“" und „komplexer Gleichheit“, die Sequenzierung und Differenzierung von „Grundbildung“ und erweiterter schulischer Bildung als interne Stufung allgemeiner schulischer Bildung vorgeschlagen wird (Tenorth 2016). Insgesamt kann er aber vor allem der Frage nicht entgehen, 
die bildungstheoretisch relevant ist, dass nämlich die Konstruktion von Bildung notwendig die Konstruktion von Differenz bedeutet, und dass deshalb auch ,nicht alle gleich viel haben“ müssen, schon gar nicht alle das Zeugnis der Hochschulreife, wie man z.B. mit Harry Frankfurt (2015) nicht nur für Besitz, sondern auch für Bildungsgüter sagen muss.

Die Argumente, die Berkemeyer im Blick auf die Kriterien von Bildungsgerechtigkeit institutionenbezogen, also für das Bildungssystem, vorträgt, nehmen solche Prämissen und Kriterien nicht hinreichend auf. Auch der pauschale Verweis auf die Arbeiten von Forst oder auf die Empirie der Bildungsdisparitäten ersetzt diese Diskussion nicht. Die Differenzierung und stufenspezifische Sequenzierung in einem Zweisäulen-System z. B. kann man nämlich durchaus als Form debattieren, wie einfache und komplexe Gleichheit im Bildungssystem zum Thema werden. Allerdings, Gleichheit im Ergebnis ist dann kein Kriterium, etwa gemessen an dem simplen Indikator der Disparitätenforschung, die den Erwerb eines Hochschulzugangszertifikats in die Rolle eines Kriteriums hebt und das mit Verweis auf „soziale Gerechtigkeit““ meint legitimieren zu können.

So naheliegend es ist, in die Analyse der Schule auch die „Kritik der gesellschaftlichen Institution“ einzubeziehen und damit „das sozialkritische Potential“ der Beobachtung des Bildungssystems zu nutzen und neben „Bildungsgerechtigkeit““ auch „soziale Gerechtigkeit“, wie bei Berkemeyer, als kriteriale Referenz hinzutreten $\mathrm{zu}$ lassen, unproblematisch und folgenlos ist diese Erweiterung nicht. Mit Walzer könnte man fragen, ob diese Vermischung der sphärenspezifischen Kriterien begründungsfähig ist, welche Folgeprobleme sie erzeugt und ob sich damit nicht das Verständnis von Bildung und Schule auch gerechtigkeitstheoretisch radikal verändert. Berkemeyer geht offenbar von einer anderen Funktion von Schule aus und eröffnet damit den dritten Diskussionsstrang, den man - um noch einmal Walzer zu bemühen - als Indiz für dessen These lesen darf, dass Pluralismus unvermeidlich ist, damit aber auch Dissens über das Verständnis der Güter, hier von Bildung und Schule: „Keine Interpretation der Bedeutung eines sozialen Guts oder der Grenzen des Bereichs, in dem es rechtmäßig seine Wirkung entfaltet (sphere within which it legitimately operates), wird unstrittig sein. Auch ist kein ordentliches Verfahren zur Hand, mit dem die unterschiedlichen Interpretationen entwickelt oder überprüft werden könnten“ (zit. nach Knoll und Spieker, S. 17). Und die Kommentatoren erläutern: „Trotz dieser Schwierigkeiten geht Walzers Ansatz von den Bedeutungen der sozialen Güter aus, die ihnen beziehungsweise ,unserem gemeinsamen Verständnis (shared understandings) " von ihnen innewohnen“" (zit. Knoll und Spieker). Am Streit über Status und Funktion der Schule, den Berkemeyer und Nerowski ebenfalls dokumentieren, kann man sehen, mit welchen Formen des Dissenses und mit welchen diskussionsbedürftigen Folgeproblemen man hier rechnen muss, wenn man über die Verteilung von „Bildung“ als eines Gutes, das im Bildungssystem konstruiert wird, gerechtigkeitstheoretisch spricht. 


\section{Bildungsgerechtigkeit angesichts von Status, Funktion und Praxis des Bildungssystems}

Das Verständnis des Bildungssystems könnte bei den Opponenten nicht unterschiedlicher sein. Nerowski geht von Schule als einem „Zweckverband“ aus, der zugleich mit der Ermöglichung von individueller Bildung auch Aufgaben der Zertifizierung und damit der folgenreichen Konstruktion von Differenz und d.h. ja auch, wie ich ergänze, eine ,symbolische Garantiefunktion“ (Nunner-Winkler) für die Gesellschaft übernimmt. Berkemeyer kritisiert das scharf, denn Schule werde damit bei Nerowski ,merkwürdig technokratisch und eindimensional gefasst und in ihren tatsächlichen Leistungen und Effekten verkannt.“ Man wird hier schon kritisch einwenden, dass Nerowski in seiner zweifachen Referenz, für das Bildungsminimum und für leistungsgerechte Beurteilung und Zertifizierung, nicht wirklich ,eindimensional“ denkt, und auch wohl kaum ,technokratisch“, wenn er im Blick auf das Subjekt und die Ermöglichung selbstbestimmter Bildungsprozesse argumentiert. All das kann man durchaus als Ausdruck der ,Zwecke“ der Organisation Schule ansehen, aber schultheoretisch ist das natürlich noch nicht genug, so wenig wie Berkemeyers Hinweis auf die schönen Zielformulierungen in Schulgesetzen und Verfassungen. Man sollte zumindest Luhmanns (1986) alte Unterscheidung von „Code“, also „,Selektion“, und „Programm“, also „Bildung“ hinzunehmen, um die Schule, mit Berkemeyer, „in ihren tatsächlichen Leistungen und Effekten“ zu sehen (und um zu sehen, dass das keineswegs eindimensional ist). Man könnte auch auf die schöne alte These verweisen, dass die „Schule als Institution erzieht“, um zu sehen, dass eine Organisation zwar zu klaren „Zwecken“ eingerichtet wird - Demokratieerziehung oder das Bildungsminimum zuerst, Zertifizierung danach -, aber dennoch, als Organisation in eigener Praxis ,multiple“ Effekte erzeugt. Es empfiehlt sich deshalb, die schulbezogenen Bemerkungen Berkemeyers selbst mit einiger Distanz zu betrachten. An einigen Exempeln seiner Argumentation will ich das abschließend tun, schon weil sie zeigen, dass die Frage der Bildungsgerechtigkeit sich nicht im Blick auf Kriterien allein lösen lässt, sondern erst in der systematisch gleichzeitigen Betrachtung von Praktiken. Dann wird Leistung als Kriterium nicht dementiert, aber die damit verbundenen Herausforderungen werden sichtbar, denen man nicht entgeht, wenn man sie einfach negiert.

Berkemeyer dagegen unterbietet die systematische Diskussion über das spezifische (schul-)pädagogische Problem der Gerechtigkeit, wenn er sich dem Thema der ,Verantwortung“ und der pädagogisch so prominenten Debatte über ,Anerkennung" zuwendet. Seine Kritik trivialisiert - anders als Nerowski - das Problem der Verantwortung als Prozessproblem pädagogischer Arbeit, denn auch wenn schulische Leistung als Ko-Konstruktion im Lehr-Lern-Prozess verstanden werden muss, wird die individuelle Zurechnung ja nicht entbehrlich, sondern ist Kern der professionellen Herausforderung. Zugleich rezipiert er die Thematik und Diskussion von „Anerkennung“ nicht in der Weise, wie sie inzwischen innerhalb der Philosophie geführt wird, z.B. in scharfer Kritik an Honneths Hegel-Lektüre (Sticker 2015), und auch nicht so, wie sie in der systematischen Diskussion der Pädagogik vorliegt. Die hat gegen den pädagogisch dominierenden, moralisierend-überhöhenden, ,leistungs"-kritischen Gebrauch des Begriffs (von Prengel bis Stojanov oder Heinrich) 
die Unbrauchbarkeit von Anerkennung als Prinzip in der Erziehungstheorie belegt, weil damit die Struktur der pädagogischen Praxis verkannt wird (so Benner 2017), und sie hat zugleich eine eindeutige Kritik an der Ignoranz der pädagogischen Anerkennungstheoretiker gegenüber dem pädagogischen Zentralproblem gezeigt, das darin besteht, dem Lernenden in seiner Leistung und Praxis ,gerecht zu werden“ (u. a. Ricken 2006, 2015). Anerkennung wird zwar als spezifisch pädagogisches Gerechtigkeitskriterium propagiert, kann diese Leistung aber weder für Schulen noch für Erziehung hinreichend erbringen - so die Kritik. Der Kritiker nimmt all diese Debatten nicht auf, meint aber dennoch, schon in der allusiven Anspielung auf gerechtigkeitstheoretische Debatten Nerowski erledigen zu können.

Seine insofern kritikanfällige Position in schultheoretischen Fragen bestätigt sich auch in weiteren Argumenten Berkemeyers. Für die Kritik an der Ungerechtigkeit der Schule als System bezieht er sich auf die „Empirie zum Thema“, die Nerowski ausgeblendet habe. Dafür nimmt er mit den selbst kontrovers diskutierten Thesen von Radtke et al. über institutionelle Diskriminierung aber Belege auf, die selbst nicht theoretisch unstrittig sind, und zusammenfassende quasi-politische Denkschriften, deren Geltung primär im politischen Konsens fundiert ist. Gerechtigkeitstheoretische Rechtfertigungen der Schule, wie sie seit Dewey vorliegen und z. B. bis zu Masschelein und Simon (2013) auch aktuell vorgetragen werden, werden vollständig ausgeblendet oder nur für das eigene Argument, verkürzt also, rezipiert, die theoretische Schwäche der Argumentation mit Disparitäten (jüngst noch Cortina und Pant 2018) kommt überhaupt nicht in den Blick.

In seinen systematischen Argumenten setzt sich diese Strategie fort. Nerowskis These von der Schule als „Zweckverband“ hält er zwar für ein ,interessantes Gedankenspiel“", aber der Gewährsmann, Miller, habe das vielleicht systematisch nicht richtig gesehen. Vor allem kritisiert er diese Qualifizierung von Schule als ,Zweckverband“ mit dem Argument ab, dass sie „multiple Ziele“ verfolge, auf Zertifizierung also nicht verkürzt werden dürfe. Sieht man davon ab, dass Nerowski das nicht tut, sondern das von Berkemeyer strapazierte Argument der Demokratieerziehung - dass ,die Fähigkeit zur demokratischen Teilhabe an der Gesellschaft das wesentlichste Ziel der Schule und Staatsziel der BRD (GG, Art. 20)“ sei - im Bildungsminimum ja selbst aufnimmt, es gehört dann schon zur Kunst der Polemik, noch diese Referenz gegen Nerowski zu wenden: „Allerdings gibt es hierfür kein Zertifikat und wäre in Nerowskis Lesart darum wohl auch unwichtig oder aber zumindest nicht nützlich. Zudem ist diese Zielerreichung konstitutiv für die Demokratie und kein Ziel, was im Kontext eines Zweckverbands frei wählbar wäre." Noch hier ignoriert er die Differenz von Bildungsminimum, das eben nicht „frei wählbar“ ist, sondern Bestandteil von Schulpflicht, und erweiterter Bildung, für die es keine Schulpflicht gibt, jedenfalls nicht für das Abitur oder die Zertifizierung (denn die Erfüllung der Schulpflicht bis zum 18. Lebensjahr wird zwar kontrolliert, schließt aber keine Zertifizierung ein, wie Jugendliche im sog. „Übergangs-System“ zu ihrem Leidwesen erfahren).

In diese wenig überlegte Argumentation Berkemeyers gehört es auch, dass er den Blick auf das im Bildungssystem etablierte System von Abschlüssen und Anschlüssen, auf das Nerowski rekurriert, mit der knappen Bemerkung abwertet, dass Nerowski, systembezogen, ,die Frage der Gerechtigkeit auf die Frage nach An- 
schlussoptionen verkürzt“. Solche schulisch erzeugten Optionen mögen für ihn unwesentlich sein, man sollte aber nicht nur erinnern, dass Bildungsarmut, stärkstes Indiz für Bildungsungerechtigkeit, außer als Kompetenzarmut auch wesentlich als Zertifikatsarmut bestimmt und in ihren Folgen analysiert wird. Nicht nur die KMK, sondern auch ein ansonsten scharfer bildungstheoretischer Kritiker der Schule macht deshalb mit guten Gründen den Grundsatz stark: „kein Abschluss ohne Anschluss" (Herrmann). Berkemeyer dagegen handelt sich politisch wie bildungstheoretisch wohl große Probleme ein, wenn er zeigen will, wie sich Anschlüsse jenseits macht- und reichtumsbasierter Willkür begründungsfähig herstellen lassen, wenn keine nachschulisch bedeutsamen schulischen Abschlüsse vorliegen.

Man kann natürlich, unbesorgt in der Frage von Abschlüssen und Anschlüssen, soziales Kapital wieder ins Recht setzen und den Zugang in Berufe oder in die tertiären Bildungsgänge von Qualifikation und Leistungsnachweisen ablösen, Bildungssystem und Beschäftigungssystem vielleicht sogar radikal entkoppeln. Dann muss man zumindest mit Wilhelm von Humboldt und der preußischen Bildungspolitik streiten, die z.B. das Abitur als verbindliches Zugangszertifikat einführten und 1834 auch gegen den Widerstand des Adels endlich generell durchgesetzt haben. Das geschah ja, um dem Leistungskriterium beim Zugang in die Universität (und in die akademischen Berufe) Geltung zu verschaffen und die Privilegien des Adels, Universitäten und akademische Berufe frei von Leistungsnachweisen besuchen zu können, endlich abzuschaffen (Blankertz 1969, S. 106 ff.). Aber, wenn man schon so wie Berkemeyer argumentiert, vielleicht setzt er ja auf das USA-Prinzip und will universitätsbasierte Zugangsregelungen (die schulische Leistung entwerten bzw. ignorieren) einführen, damit es gerecht zugeht. Dann haben wenigstens, wie man wissen kann (Karabel 2005), auch ,the stupid sons of the rich“ wieder die legitime Chance zum Universitätsbesuch, denn für sie sorgt bis heute zwar ohne Abschluss und Leistungsnachweis, aber sehr verlässlich z. B. das Alumni-System, das die Anschlüsse schon herstellt - in gerechter Weise?

Berkemeyer greift solche Debatten der Folgeprobleme seiner im Lichte schon der historischen Erfahrung leichtfertigen Kritik von schulischer Zertifizierung und Leistungsbeurteilung nicht auf, sondern bezieht sich allein auf das „Äquivalenzprinzip“, das im Bildungssystem nicht gewahrt werde, sichtbar an der Ungleichheit und Ungleichwertigkeit der Abiturzeugnisse. Das trifft zu, wie jüngst ja auch noch das Bundesverfassungsgericht gerügt hat, gestützt auf die empirische Schulforschung. Aber das B VerfG hat deshalb Schulnoten oder das Abitur weder als Zugangskriterien aufgehoben noch ihre Legitimation grundsätzlich bezweifelt, allerdings Nachbesserung in den Praktiken der Bewertung verlangt und neue, ebenfalls leistungsbasierte Verfahren gefordert. Auch alternative Zugangswege in die Hochschule setzen ja nicht zufällig Abschlüsse und zertifizierte Erfahrung voraus, wie z.B. beim Zugang ohne Abitur im Meisterstudium (etc.).

Hier kommen also die Praktiken zu ihrem Recht und die professionelle Kompetenz der Pädagogen, auch bewertend bis zur Zertifizierung ihren Adressaten gerecht zu werden. Erst dann wird auch die komplexe Aufgabe der Schule angemessen gerechtigkeitstheoretisch gesehen und nicht in Kritik oder in Reformrhetorik unterboten. Für die Frage, wie es gelingt, die Vielfalt der Erwartungen an Schule innersystemisch und in der Interaktion zu prozedieren, und auch die dabei unver- 
meidliche Konstruktion von Differenz und d.h. auch von legitimer Ungleichheit, orientiert an universalen Kriterien und mit gesellschaftlicher Relevanz pädagogisch verantwortlich und legitim zu praktizieren, sind dann letztlich beide Autoren nicht mehr aussagekräftig. Nerowski erinnert zumindest noch daran, dass Leistung als Kriterium im Interesse von Individuum und Gesellschaft nicht verzichtbar ist; Berkemeyer verweist hier und da auf die Frage der „Praktiken“, begnügt sich ansonsten aber mit einer Kritik, die ihrer eigenen Implikationen und der systematischen Referenzen, die sie beansprucht, nicht gewiss ist. Die Debatte über die Handhabung von Leistung und ihre individuelle Zurechnung in Schulen ist deshalb weder mit Nerowskis Beitrag noch mit Berkemeyers Art von Kritik beendet, sondern bestenfalls eröffnet.

Funding Open Access funding provided by Projekt DEAL.

Open Access Dieser Artikel wird unter der Creative Commons Namensnennung 4.0 International Lizenz veröffentlicht, welche die Nutzung, Vervielfältigung, Bearbeitung, Verbreitung und Wiedergabe in jeglichem Medium und Format erlaubt, sofern Sie den/die ursprünglichen Autor(en) und die Quelle ordnungsgemäß nennen, einen Link zur Creative Commons Lizenz beifügen und angeben, ob Änderungen vorgenommen wurden.

Die in diesem Artikel enthaltenen Bilder und sonstiges Drittmaterial unterliegen ebenfalls der genannten Creative Commons Lizenz, sofern sich aus der Abbildungslegende nichts anderes ergibt. Sofern das betreffende Material nicht unter der genannten Creative Commons Lizenz steht und die betreffende Handlung nicht nach gesetzlichen Vorschriften erlaubt ist, ist für die oben aufgeführten Weiterverwendungen des Materials die Einwilligung des jeweiligen Rechteinhabers einzuholen.

Weitere Details zur Lizenz entnehmen Sie bitte der Lizenzinformation auf http://creativecommons.org/ licenses/by/4.0/deed.de.

\section{Literatur}

Benner, D. (2017). Über Anerkennung und Macht in pädagogischen Kontexten. In I.C. Thompson \& S. Schenk (Hrsg.), Zwischenwelten der Pädagogik. Festschrift für Alfred Schäfer (S. 139-153). Paderborn: Schöningh.

Berkemeyer, N. (2018). Über die Schwierigkeit, das Leistungsprinzip im Schulsystem gerechtigkeitstheoretisch zu begründen. Replik auf Christian Nerowski. Zeitschrift für Erziehungswissenschaft, 21, $447-464$.

Blankertz, H. (1969). Bildung im Zeitalter der großen Industrie. Pädagogik, Schule und Berufsbildung im 19. Jahrhundert. Hannover: Schroedel.

Bohnsack, F. (2013). Wie Schüler die Schule erleben. Zur Bedeutung der Anerkennung, der Bestätigung und der Akzeptanz von Schwäche. Opladen: Budrich.

Cortina, K. S., \& Pant, H. A. (2018). Ignorierte Differenzen, illegitime Disparitäten - Über Betriebslindheit im Disparitätendiskurs der empirischen Bildungsforschung. Zeitschrift für Pädagogik, 64, 71-79.

Frankfurt, H. (2015). Ungleichheit. Warum wir nicht alle gleich viel haben müssen. Berlin: Suhrkamp.

Herrmann, U. (2004). Alternativen zum Schwindel mit den „Bildungsstandards“. Ein Zehn-Punkte-Programm. Die Deutsche Schule, 96, 134-137.

Herrmann, U. (2011). Fördern „Bildungsstandards“ die allgemeine Schulbildung? https://evangelischerschulbund-nord.de/wp-content/uploads/2011/10/Bildungsstandards.Mnster.pdf. Zugegriffen: 15. Jan. 2019.

Karabel, J. (2005). The chosen. The hidden history of admission and exclusion at Harvard, Yale and Princeton. Boston: Houghton-Mifflin.

Klafki, W. (1991). Sinn und Unsinn des Leistungsprinzips in der Erziehung. In W. Klafki (Hrsg.), Neue Studien zur Bildungstheorie und Didaktik (2. Aufl., S. 209-247). Weinheim: Beltz. 
Knoll, M., \& Spieker, M. (2014). Einführung in Walzers Gerechtigkeitstheorie. In M. Knoll \& M. Spieker (Hrsg.), Michael Walzer. Sphären der Gerechtigkeit. Ein kooperativer Kommentar. Stuttgart: Steiner.

Luhmann, N. (1986). Codierung und Programmierung. Bildung und Selektion im Erziehungssystem. In H.E. Tenorth (Hrsg.), Allgemeine Bildung. Analysen zu ihrer Wirklichkeit, Versuche über ihre Zukunft (S. 154-182). Weinheim: Juventa.

Manitius, V., Hermstein, B., Berkemeyer, N., \& Bos, W. (Hrsg.). (2015). Zur Gerechtigkeit von Schule. Theorien, Konzepte, Analysen. Münster: Waxmann.

Masschelein, J., \& Simons, M. (2013). In defence of the school. A public issue. Leuven: E-ducation, Culture $\&$ Society Publishers.

Mollenhauer, K. (1964). Pädagogik und Rationalität. In K. Mollenhauer (Hrsg.), Erziehung und Emanzipation (S. 55-74). München: Juventa.

Nerowski, C. (2018). Leistung als Kriterium von Bildungsgerechtigkeit. Zeitschrift für Erziehungswissenschaft, 21(3), 441-464.

Ricken, N. (2006). Erziehung und Anerkennung. Zur Konstitution des pädagogischen Problems. Vierteljahrsschrift für wissenschaftliche Pädagogik, 82, 215-230.

Ricken, N. (2015). Was heißt ,,jemandem gerecht werden“? Zum Problem der Anerkennungsgerechtigkeit im Kontext von Bildungsgerechtigkeit. In V. Manitius, B. Hermstein, N. Berkemeyer \& W. Bos (Hrsg.), Zur Gerechtigkeit von Schule: Theorien, Konzepte, Analysen (S. 131-149). Münster: Waxmann.

Schwaabe, C. (2014). Erziehung und Bildung bei Michael Walzer: Schule der Gerechtigkeit? In M. Knoll \& M. Spieker (Hrsg.), Michael Walzer. Sphären der Gerechtigkeit. Ein kooperativer Kommentar (S. 177-190). Stuttgart: Steiner.

Sticker, M. (2015). Hegels Kritik der Anerkennungsphilosophie. Die Aufhebung verwirklichter Anerkennung in der Phänomenologie des Geistes. Hegel-Studien, 49, 89-122.

Tenorth, H.-E. (2016). Bildungstheorie und Bildungsforschung, Bildung und kulturelle Basiskompetenzen - Ein Klärungsversuch, auch am Beispiel der PISA-Studien. In J. Baumert \& K.-J. Tillmann (Hrsg.), Empirische Bildungsforschung. Der kritische Blick und die Antwort auf die Kritiker. Zeitschrift für Erziehungswissenschaft: Sonderheft 19. (S. 45-71). Wiesbaden: Springer VS.

Thurn, S. (2004). Leistung - was ist das eigentlich? Oder: „Die Würde des heranwachsenden Menschen macht aus, sein eigener ,Standard" sein zu dürfen“. Neue Sammlung, 44, 419-435.

Walzer, M. (1983). Spheres of justice. A defense of pluralism and equality. New York: Basic Books.

Walzer, M. (2006). Sphären der Gerechtigkeit. Ein Plädoyer für Pluralität und Gleichheit. übers. von Hanne Herkommer. Frankfurt a. M.: Campus.

Wigger, L. (2015). Bildung und Gerechtigkeit - Eine Kritik des Diskurses um Bildungsgerechtigkeit aus bildungstheoretischer Sicht. In V. Manitius, B. Hermstein, N. Berkemeyer \& W. Bos (Hrsg.), Zur Gerechtigkeit von Schule (S. 72-92). Münster: Waxmann. 